\title{
Drug utilization review of ciprofloxacin in the outpatient department of Boru Meda Hospital, South Wollo Zone, Amhara Region, Ethiopia
}

\author{
Tessema Tsehay Biru ${ }^{1}$, Amsalu Degu Defersha ${ }^{2}$, Belayneh Kefale Gelaw ${ }^{2}$, \\ Gobezie Temesgen Tegegne ${ }^{2}$
}

${ }^{1}$ Department of Pharmacy, College of Health Sciences, Mizan Tepi University, SNNP region, Ethiopia, ${ }^{2}$ Department of Pharmacy, College of Medicine and Health Science, Ambo University, Oromia region, Ethiopia

Received: 13 December 2013 Accepted: 27 December 2013

*Correspondence to:

Tessema Tsehay Biru,

Email: tessema.tsehay@gmail.com

(C) 2014 Biru TT et al. This is an open-access article distributed under the terms of the Creative Commons Attribution NonCommercial License, which permits unrestricted noncommercial use, distribution, and reproduction in any medium, provided the original work is properly cited.

\begin{abstract}
Background: The antimicrobial agents developed during the last 60 years are among the most dramatic examples of the advances of modern medicine. Many infectious diseases once considered incurable and lethal are now amenable to treatment with a few pills. They are among the most frequently prescribed medications to day although microbial resistance due to evolutionary and misuse threatens their continued efficacy. One mechanism to ensure correct prescribing and use is drug use evaluations studies. Objective of current study is to evaluate retrospectively of ciprofloxacin use from outpatient medical records by using pre- set criteria in Boru Meda hospital.

Methods: A cross-sectional study on retrospectively collected data was used to evaluate ciprofloxacin use from outpatient medical history records existing from January 18, 2009 to January 17, 2010. Criteria along with thresholds set by experts for undertaking drug use evaluation for ciprofloxacin was used which is modified based on Ethiopian National Drug Formulary and Ethiopian National Standard Treatment Guideline, which are used in the hospital. Data was collected from January 18 to January 25, 2010 using appropriate data collection format.
\end{abstract}

Results: Twenty six $(65 \%)$ patients were in the age group of 15 to 49 years and twenty $(50 \%)$ were females. Thirty eight $(95 \%)$ and each of thirty seven $(92.5 \%)$ cases were consistent with the Ethiopian National Standard Treatment Guideline \& drug formulary regarding indication, dose \& frequency of ciprofloxacin use in the outpatient department of Boru Meda hospital. 47.5\% ciprofloxacin use has problem in duration of treatment. There were potential drug interactions with the drug in $40 \%$ of the cases. $10 \%$ of ciprofloxacin use was against contraindication.

Conclusions: Due From the retrospective Drug Use Evaluation (DUE) study, it was identified that there was inappropriate ciprofloxacin use in the outpatient department of Boru Meda hospital even though the drug's use regarding indications was a better performance and dosing practices were almost appropriate as per the criteria used for the study (assuming that there were no dose adjustments). There was a great problem concerning the duration of ciprofloxacin drug therapy. Ciprofloxacin use along with potentially interacting drugs and against contraindications was also another problem indicated in the study.

Keywords: Drug use evaluation, BMH, STG, Ciprofloxacin, Boru Meda

\section{INTRODUCTION}

Antibiotics are microbial metabolites or synthetic analogs inspired by them that, in small doses, inhibit the growth and survival of microorganisms without serious toxicity to the host. Empirical therapy, definitive therapy, and prophylactic or preventive therapy are possible with these agents. Many infectious diseases once considered incurable and lethal are now amenable to treatment with a few pills. Antibiotics are among the most frequently prescribed medications today although microbial resistance due to evolutionary pressures and misuse 
threatens their continued efficacy. Unfortunately we cannot any longer confidently depend upon the discovery of an increasing numbers of novel antibiotics and antimicrobial agents to keep infectious diseases under control but must increasingly pay attention to neglected public health measures and concentrate upon using antibiotics safely and effectively. ${ }^{1-3}$

One of the most pressing problems facing public health providers and administrators in many countries is ensuring rational use of drugs. The conference of Experts on the Rational Use of Drugs, convened by the World Health Organization (WHO) in Nairobi in 1985 defined rational drug use as "the rational use of drugs requires that patients receive medications appropriate to their clinical needs, in doses that meet their own individual requirements, for an adequate period of time and at the lowest cost to them and their community. ${ }^{4}$

Rational drug use implies an individual approach to patient treatment .The presence of standard treatment guidelines and drug formularies for selected drugs in a health facility does not ensure that they are prescribed and used correctly. One mechanism to ensure correct prescribing and use is drug use evaluation (DUE). ${ }^{4}$

DUE is a performance improvement method that focuses on evaluating and improving drug use processes to achieve optimal patient outcomes. DUE may be applied to a drug or therapeutic class, or diagnosis. Through its focus on the system of drug use, DUE helps to identify actual and potential drug -drug related problems that could interfere with achieving optimum outcomes from drug therapy. DUE is of three types; prospective, concurrent and retrospective. 5,6

A retrospective DUE is the simplest to perform since drugs therapy is reviewed after patient has received the medication. A retrospective review may detect patterns in prescribing, dispensing or administering drugs to prevent recurrence of inappropriate use and serves as a means for developing prospective standards and target interventions. In retrospective DUE, patient medical charts or computerized records are screened to determine whether the drug therapy met approved criteria and aids prescribers in improving care for their patients, individually and within groups of patients. ${ }^{6}$

Quinolones are groups of antimicrobial drugs having a wide clinical application. The introduction of fluoroquinolones such as ciprofloxacin represents a particularly important therapeutic advance because these agents have broad antimicrobial activity and are effective after oral administration for the treatment of a wide variety of infectious diseases. Antimicrobial prophylaxis often with an oral fluoroquinolones is used to prevent a variety of infections in patients undergoing organ transplantation or receiving cancer chemotherapy. ${ }^{2}$
Ciprofloxacin, being second generation fluoroquinolones, has wide clinical importance. It is the most frequently used fluoroquinolone in the United States and also included in the National Drug List of Ethiopia to be used for a variety of bacterial infections. It has paramount importance in treating infections caused by many Enterobacteriaceae and other gram negative bacilli. Ciprofloxacin is the most potent of fluoroquinolones for pseudomonal infections associated with cystic fibrosis. It is the drug of choice for prophylaxis and treatment of anthrax. ${ }^{7,8}$

\section{Statement of the problem}

Although antimicrobial drugs are among the safest and least toxic of drugs used in medical practice, they are also included under the most commonly misused of all drugs. Excessive amounts can result in significant toxicities especially in patients with impaired drug excretion or metabolism. The use of too low a dose may result in treatment failure and is most likely to select for microbial resistance. Hence dosing errors which can be the wrong frequency of administration or the use of either an excessive or sub-therapeutic dose are common in using antimicrobial agents. $^{2}$

The development of bacterial resistance of antibiotics has become a major problem throughout the world. Resistant organisms may emerge as a result of many factors, including irrational use of drugs one form of which is irrational prescribing practice. Studies have shown that $22-65 \%$ of antibiotic prescriptions are inappropriate. ${ }^{9-11}$

An inevitable consequence of antimicrobial usage is the selection of resistant microorganisms. Overuse \& inappropriate use of antibiotics has fueled a major increase in prevalence of multidrug resistant pathogens, leading some to speculate that we are nearing the end of the antibiotic era. ${ }^{3}$

Fluoroquinolones are extremely useful agents and an important therapeutic advance. They are relatively nontoxic, well tolerated, broad spectrum agents. Their excellent oral bioavailability permits their use for treatment of variety of serious bacterial infections such as those caused by Pseudomonas aeruginosa or Methicillin Resistant Staphylococcus aureus (MRSA), which formerly could be treated only with parental agents. As strains of $E$.coli and other gram negative pathogens resistant to trimethoprim-sulfamethoxazole have become more common, fluoroquinolones are increasingly relied on for empirical therapy of urinary tract infections. ${ }^{3}$

Unfortunately overuse and inappropriate use have already eroded the clinical utility of fluoroquinolones. Overuse of the fluoroquinolones particularly in the hospital settings, has led to selection of resistance and this resistance has increased after their introduction into clinical practice especially in Pseudomonas and Staphylococci. ${ }^{2,3}$ 
Studies on ciprofloxacin in different places showed that; in 2004 the prevalence of ciprofloxacin resistance was $7 \%$ in Cape Town and $11 \%$ in Johannesburg. In 2007, 37/139 (27\%) Cape Town isolates, 47/149 (32\%) Johannesburg isolates were resistant to ciprofloxacin in comparison with 2004 data; this represents 2-9 fold and 1.9 fold increase respectively. These increases, in part, associated with over use of ciprofloxacin. ${ }^{12}$

\section{Significance of the study}

The study design to evaluate the use of ciprofloxacin in Boru Meda hospital to provide an over view ciprofloxacin use in hospital and to promote the rational prescribing, dispensing, and administration of ciprofloxacin. Hence to reduce the emergence of antibiotic resistance. More over the study contributes in identifying medication related problems and areas of in appropriate use by that it helps in identifying areas in which further information and education may be needed by health care providers.

Furthermore, the study helps for policy makers, baseline for other studies, guides other health care providers to prescribe drugs correctly.

\section{General Objective}

To evaluate retrospectively of ciprofloxacin use from outpatient medical records by using pre-set criteria in Boru Meda hospital

\section{Specific Objectives}

- To evaluate whether ciprofloxacin is appropriately indicated or not

- To evaluate whether the dosage regimen (dose, frequency, duration) is in accordance with guidelines used in the hospital

- To assess any drug -drug interactions with concomitantly administered drugs

- To assess extent of contraindications

\section{METHODS}

\section{Study area and period}

The study was conducted in Boru Meda hospital which is found in south wollo zone, Amhara region. It is located $410 \mathrm{kms}$ far from Addis Ababa and only 10 kilometers from Dessie town which is the center of the zone. There are 3 private \& 3 governmental hospitals in south wollo zone, Boru Meda hospital being one of the governmental hospitals. There are 42 health professionals working in the hospital: 1 specialist, 3 general practitioners \& 2 pharmacists among others. The study was conducted from January 18 to January 25, 2010.

\section{Study Design}

A cross sectional, retrospective study was done to evaluate ciprofloxacin use using a pre -set criteria along with threshold which was set based on the National STG $\&$ formulary used in the hospital. Patient medical history cards existing from January 18, 2009 to January 17, 2010 was evaluated against the pre-set criteria using appropriate data collection format.

\section{Population}

Source population

All the patient medical history cards in Boru Meda hospital during the period of January 18, 2009 to January 17,2010 .

\section{Study population}

All medical history cards of outpatients containing ciprofloxacin in Boru Meda hospital during the period of January 18, 2009 to January 17, 2010.

\section{Sample population}

The sample population was determined depending on drug use evaluation guideline.

\section{Sample size}

The size of the sample population was determined according to the Joint Commission on the Accreditation of Health care Organization (JCAHO) criteria which mandates a sample size of: $5 \%$ If the average number of cases per quarter is greater than 600 and at least 30 , if quarter cases are less than 600. From the study which encompasses 1 year data of ciprofloxacin use in the outpatient department of Boru Meda hospital, the number of cases (ciprofloxacin indications) per quarter was found to be 204(a total of 815 annual cases). Therefore 40 was the size of sample in the study. ${ }^{19}$

\section{Sampling technique}

40 outpatient medical history cards were selected using systematic random sampling method from sequentially arranged cards (by date of ciprofloxacin indication) of outpatients that were on ciprofloxacin ( $\mathrm{Cfx}$ ) in the hospital during the period of January 18, 2009 to January 17, 2010. Every $20^{\text {th }}$ card was taken as a sample population (sampling interval=total source population/sample size $=>815 / 40=20$ ).

\section{Study variables}

\section{Independent variables}

- Patient characteristics: age, sex, pregnancy, lactation

- Diagnosis: qualification of prescribers 


\section{Dependent variables}

- Indication

- Dosage regimen (dose, frequency, duration)

- Drug-drug interaction

- Contraindication

\section{Data quality control}

Data collection format containing the variables to be measured was developed and used. Maximum care was taken for the quality of the data to be collected.

\section{Data Collection}

Obtaining permission to conduct the study, the data was collected using a pre-tested data collection format by the principal investigator.

\section{Data processing and Analysis}

The collected data was manually sorted, categorized and then results were evaluated against the set criteria and thresholds, and presented in tables.

\section{Pre- test}

The data collection format was pre-tested for its validity, reliability and consistency.

\section{Ethical consideration}

Prior to data collection, formal letter was written from Jimma University Student Research Program to Boru Meda hospital so as to conduct the study.

\section{Limitation of the study}

Unavailability of adequate literature on the subject matter of the study \& poor patient medical history taking practice (especially drug history) were worth mentioning as limitations to prepare this research paper.

\section{RESULTS}

\section{Age and sex distribution of patients with ciprofloxacin indication}

A total of 40 outpatient medical history cards indicated with ciprofloxacin were included in the study. Out of 40 patients, $20(50 \%)$ were males and $20(50 \%)$ were females who were neither pregnant nor lactating. 3, 26 and 11 of the patients were in the age ranges of $<15$ years, 15-49 years and $>50$ years respectively (Table 1 ).

\section{Indications of ciprofloxacin}

Ciprofloxacin was indicated for a variety of disease conditions. $85 \%$ of ciprofloxacin used was indicated for the treatment of Acute Gastro Enteritis (AGE, 30\%), Urinary Tract Infections (UTIs, 30\%), Acute Febrile Illnesses (AFIs, 15\%) and bacterial conjunctivitis (10\%) (Figure 1).

Table 1: Age and sex distribution of patients with ciprofloxacin indication in the outpatient department of Boru Meda hospital, January 18, 2009 to January 17, 2010.

\begin{tabular}{|ll|}
\hline Variable & Frequency $(\%)$ \\
\hline Age $($ Years) & \\
\hline$<15$ & $3(7.5)$ \\
\hline $15-49$ & $26(65)$ \\
\hline$>50$ & $51(27.5)$ \\
\hline Total & $40(100)$ \\
\hline Sex & \\
\hline Male & $20(50)$ \\
\hline Female & $20(50)$ \\
\hline Total & $40(100)$ \\
\hline
\end{tabular}

\section{Indication Appropriateness of ciprofloxacin}

Thirty eight (95\%) ciprofloxacin indications were appropriate based on Ethiopian National Drug Formulary (2004 edition) used in the hospital. Only 2(5\%) indications were not in line with the formulary which was indications for varicose vein and intestinal parasite infections.

\section{Dosage regimen (dose, frequency, duration) of ciprofloxacin}

It was found that out of 40 outpatient medical history cards, $37(92.5 \%)$ contained correct ciprofloxacin dosing while $3(7.5 \%)$ cards contained over dosing. There were no under dosing regimens. $37(92.5 \%)$ indications were with correct frequency of administration while $3(7.5 \%)$ with incorrect frequency. $21(52.5 \%), 18(45 \%)$ and 1 $(2.5 \%)$ cards contained correct, long and short duration of treatment respectively (Table 2 ).

\section{Drug interactions with ciprofloxacin}

Forty percent (16) of cards contained one or more potentially interacting drug with ciprofloxacin. Paracetamol, diclofenac, and antacids accounted 7 $(17.5 \% 0,7(17.5 \%)$ and $2(5 \%)$ of the interacting drugs respectively.

\section{Ciprofloxacin use against contraindications}

There were no pregnant and lactating patients for whom ciprofloxacin was indicated in the study, but it was indicated for four $(10 \%)$ children whose ages were $<18$ years which is against contraindications. 


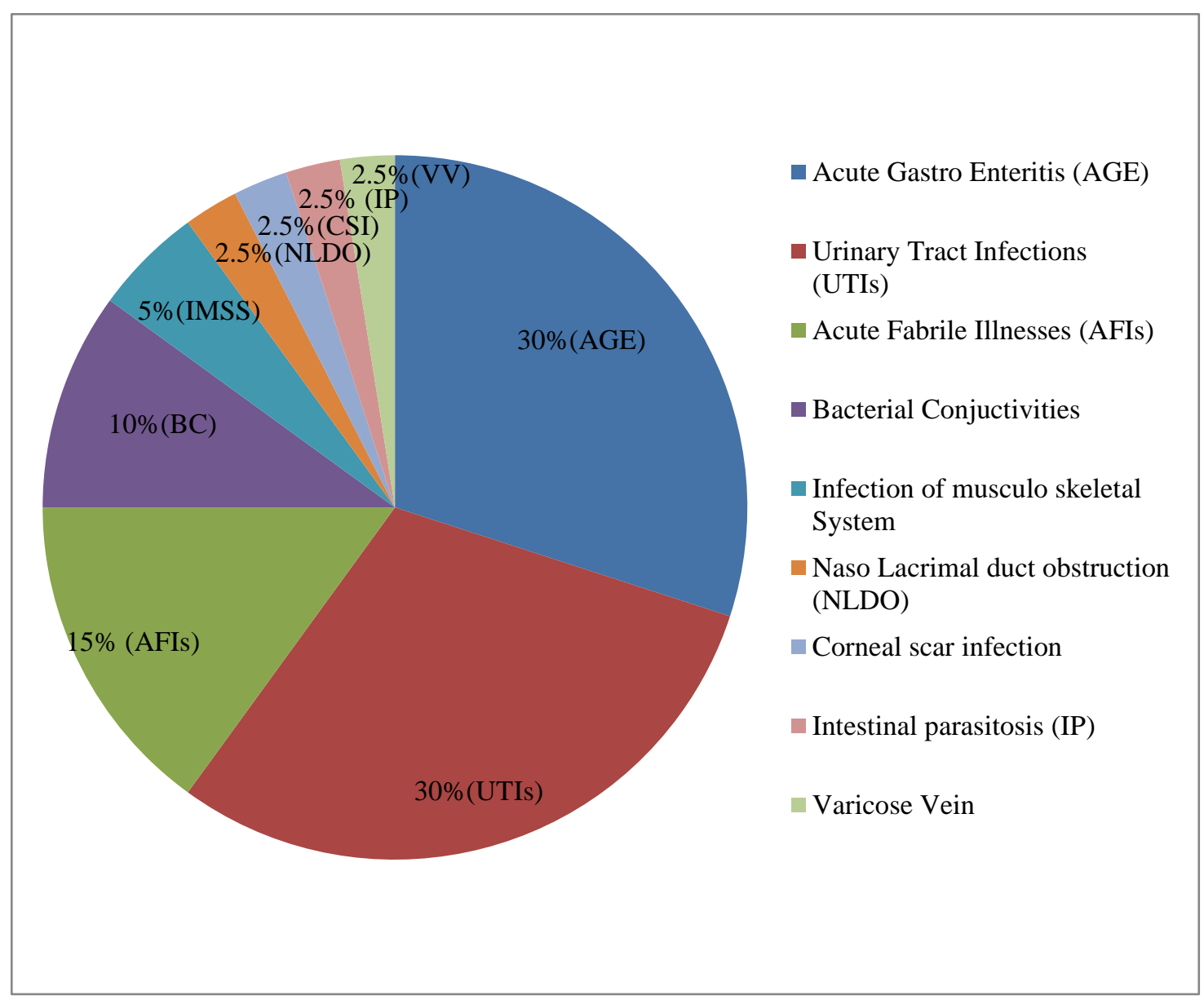

Figure 1: Disease conditions for using ciprofloxacin in the outpatient department of BMH, January 18, 2009 to January 17, 2010.

Table 2: Dosage regimen (dose, frequency, duration) of ciprofloxacin in the outpatient department of Boru Meda hospital, January 18, 2009 to January 17, 2010.

\begin{tabular}{|c|c|c|c|c|c|c|c|}
\hline \multirow{2}{*}{ Indicators } & \multirow{2}{*}{ Variable } & \multirow{2}{*}{ Frequency $(\%)$} & \multicolumn{2}{|l|}{ Sex } & \multicolumn{3}{|c|}{ Age (years) } \\
\hline & & & Male & Female & $<15$ & $15-49$ & $>50$ \\
\hline \multirow[t]{4}{*}{ Dose } & Correct dose & $37(92.5)$ & 19 & 18 & 3 & 24 & 10 \\
\hline & Under dose & - & - & - & - & - & - \\
\hline & Over dose & $3(7.5)$ & 1 & 2 & - & 2 & 1 \\
\hline & Total & $40(100)$ & 20 & 20 & & 2 & 1 \\
\hline \multirow[t]{3}{*}{ Frequency } & Correct frequency & $37(92.5)$ & 19 & 18 & 3 & 23 & 11 \\
\hline & Incorrect frequency & $3(7.5)$ & 1 & 2 & - & 3 & - \\
\hline & Total & $40(100)$ & 20 & 20 & 3 & 26 & 11 \\
\hline \multirow[t]{4}{*}{ Duration } & Correct duration & $21(52.5)$ & 12 & 9 & 3 & 11 & 7 \\
\hline & Short duration & $1(2.5)$ & - & 1 & - & 1 & - \\
\hline & Long duration & $18(45)$ & 8 & 10 & - & 14 & 4 \\
\hline & Total & $40(100)$ & 20 & 20 & 3 & 26 & 11 \\
\hline
\end{tabular}

\section{Actual performance versus set criteria \& thresholds for ciprofloxacin}

From the study, it was found that $95 \%$ of ciprofloxacin indications were appropriate, $92.5 \%$ and $52.5 \%$ of indications contained correct doing and duration of the drug respectively, and $60 \%$ of the cards were free from potential drug interactions while $90 \%$ of ciprofloxacin use was not against contraindications (Table 3 ). 
Table 3: Actual performance versus set criteria and thresholds for ciprofloxacin in the outpatient department of Boru Meda Hospital, January 18, 2009 to January 17, 2010.

\begin{tabular}{|lll|}
\hline Criteria & $\begin{array}{l}\text { Expectation / } \\
\text { Threshold } \\
\text { No }(\%)\end{array}$ & $\begin{array}{l}\text { Actual } \\
\text { performance } \\
\text { No }(\%)\end{array}$ \\
\hline Indication & $36(90)$ & $38(95)$ \\
\hline Dose & $38(95)$ & $37(92.5)$ \\
\hline Duration & $38(95)$ & $21(52.5)$ \\
\hline Drug interaction & $36(90)$ & $24(60)$ \\
\hline Contraindication & $40(100)$ & $36(90)$ \\
\hline
\end{tabular}

\section{DISCUSSION}

Diabetes The purpose of Drug Use Evaluation is to ensure that drugs are used appropriately, safely and effectively to improve patient health status. In addition, continual improvement in the appropriate and effective use of drugs has the potential to lower the overall cost of care. ${ }^{6}$

Success of treatment largely depends on the ability of a physician to diagnose the major health problems (s) of a patient, select the correct drug, dosage form and route of administration, foresee probable adverse reactions and drug interactions, and prevent unnecessary or dangerous duplication of therapy. Implementation of hospital drug formulary can be considered the basis of rational drug use. However the existence of a rationally derived list of drugs approved for procurement and use in a hospital does not ensure that they are prescribed and used correctly. ${ }^{4}$

Drugs have to be prescribed (indicated) appropriately, on the basis of clinical diseases identified through diagnosis. Hence, prescribers should stick with standard treatment guidelines (STGs) while prescribing any drug. Regarding to the indications of ciprofloxacin, the study showed that $95 \%$ of the indications were appropriate as per the national drug formulary which is even a better performance as compared with the threshold set for indication of ciprofloxacin $(90 \%)$. The result is almost similar to a retrospective study done in Cleveland, veterans Administration Medical Center, which revealed that $100 \%$ ciprofloxacin uses were appropriate. ${ }^{14}$

Different doses of ciprofloxacin are used for a variety of infections and age groups. Sub clinical use of the drug results in ineffective control of infectious diseases while overdose results in toxicity problems. Therefore optimal dose has to be used for optimal treatment out comes. $92.5 \%$ correct dose \& $7.5 \%$ overdose practices were revealed in the study (assuming that there were no dose adjustments). There were no under dosing of ciprofloxacin identified. This is more than the percentage revealed from a retrospective study conducted in Central North Carolina, in 10 community nursing facilities which showed that, out of 95 regimens ,only $81 \%$ ciprofloxacin dosing were correct. $19 \%$ regimens contained inappropriate dosing of which $5.7 \%$ regimens with potentially suboptimal dosing (under dose) while $13.3 \%$ doing were over the appropriate (optimal) dose (over dose). ${ }^{13}$

Drug resistance has become a great problem associated with the use of antimicrobial agents. Prolonged use of ciprofloxacin, one of the antimicrobial agents can lead to emergence of microbial drug resistance. Hence it should be used appropriately according to the duration of treatment needed with the particular disease available in STGs and formularies. Inappropriate duration of therapy was the major problem revealed by the study in the study hospital. Only $52.5 \%$ ciprofloxacin uses were with correct duration of therapy which is very far from the threshold set for ciprofloxacin duration of therapy (95\%) . $2.5 \%$ of the indications were with short treatment periods while long treatment periods accounted for $45 \%$ of indications which can lead to development of resistance . This result showed less percentage of correct duration as compared with a retrospective study done in central North Carolina which revealed only $8 \%$ excessive (long) duration of therapy. ${ }^{3,13}$

The simultaneous use of two or more drugs is recommended in specifically defined situations based on pharmacological rational. However, selection of an appropriate combination requires an understanding of the potential for interaction between the drugs. Interactions may, otherwise, affect the patient negatively, from the study; it was found that $40 \%$ of ciprofloxacin use had one or more potentially interacting drug of which paracetamol $\&$ diclofenac (Analgesics) each accounted $43.75 \%$ and antacids $12.5 \%$ of the interacting drugs. Coadministration of ciprofloxacin with analgesics may cause convulsion. Absorption of the drug may be interfered with antacid containing $\mathrm{Al}$ or $\mathrm{Mg}$. Drug interaction results are different from the threshold set for drug interaction with ciprofloxacin $(90 \%)$. This difference might be due to the practice of prescribing multiple drugs together. ${ }^{2}$

Prescribing drugs against contraindications should be avoided unless the benefit of doing so outweighs the risk. Ciprofloxacin was used despite contraindications in $10 \%$ of the cases, children under 18 years old. The pregnancy and lactation history of female patients who were in the fertile age group $(60 \%)$ and history of patients with known hypersensitivity reaction to ciprofloxacin and other quinolones were not documented. This might imply that the drug may have been used in such type of patients for whom ciprofloxacin use is a contraindication practice. The result is different from the threshold set for contraindication use with ciprofloxacin which mandates $0 \%$ use against contraindications (a threshold value of $100 \%)$.

To date, there are no published studies conducted on retrospective DUE of ciprofloxacin in Ethiopia, hence it was not possible to compare the practice of Boru Meda 
hospital with practices of other clinical settings in Ethiopia. Also due to unavailability of adequate relevant literature to the topic, the findings were discussed mainly in relation to ciprofloxacin use criteria cited in the 2004 national drug formulary and national STG of Ethiopia which were found in the hospital to guide safe, effective and appropriate drug therapy .

\section{CONCLUSION}

From the retrospective Drug Use Evaluation study, it was identified that there was inappropriate ciprofloxacin use in the outpatient department of Boru Meda Hospital even though the drug's use regarding indications was a better performance and dosing practices were almost appropriate as per the criteria used for the study(assuming that there were no dose adjustments). There was a great problem concerning the duration of ciprofloxacin drug therapy. Ciprofloxacin use along with potentially interacting drugs and against contraindications was also another problem indicated in the study.

\section{Recommendations}

- If the gains from appropriate drug therapy are needed to be maximized, prescribers should stick with standard treatment guidelines while prescribing drugs

- Regular Drug Use Evaluation studies have to be undertaken in clinical settings where multiple drugs are prescribed so that appropriate, safe \& cost effective drug therapy can be given to patients.

- It is important for health facilities to arrange trainings on rational prescribing practice to prescribers.

\section{ACKNOWLEDGEMENTS}

We are very grateful to our college staff members for unreserved guidance and constructive suggestions and comments from the stage of proposal development to this end. We would like to thank Jimma University for supporting the budget which required for this research. Finally our deepest gratitude goes to Boru Meda Hospital staff workers who help and allow us in collecting and gathering data from the hospital.

\section{Acronyms and Abbreviations}

BID- Twice a day

BMH- Boru Meda Hospital

CFX-Ciprofloxacin

DUE- Drug Use Evaluation

DUR- Drug Use Review

GI- Gastro Intestinal

HIV/AIDS- Human Immuno Deficiency Virus / Acquired Immuno Deficiency Syndrome

IV- Intra Venous

MRSA- Methicillin Resistant Staphylococcus Aureus

OPD- Out Patient Department

PI- Principal Investigator

RTIs- Respiratory Tract Infections
STG- Standard Treatment Guideline

UTIs- Urinary Tract Infections

VAMCC- Veterans Administration Medical Center, Cleveland

WHO- World Health Organization

Funding: Jimma University

Conflict of interest: None declared

Ethical approval: Approval and permission was sought from Ethical Review Board of College of Medicine and Health Sciences of Jimma University

\section{REFERENCES}

1. Lester AM. Antibiotic and antimicrobial agents. In: Foyes principles of Medicinal chemistry, Lippincott Williams and Wilkins. $5^{\text {th }}$ ed. Philadelphia, USA: Wolters Kluwer; 2002: 819 and 825.

2. Henry F. Chambers. General considerations of antimicrobial therapy. In: Laurence B. Burtol, Jones L, Keithl, Parker: Goodman and Gilman's pharmacologic basis of therapeutics. $11^{\text {th }}$ ed. New York: McGraw-Hill; 2006: 1095 -1119.

3. Bertram G. Katzung. Basic and clinical pharmacology. $7^{\text {th }}$ ed. USA: McGraw-Hill publishers; 1998: 723 and 767.

4. Moore T, Bykov A, Savelli T, Zagorski A: Guidelines for implementing drug utilization review programs in hospitals, Management Sciences for Health, Arlington VA / Moscow, Russia, 1997. Available http://pdf.usaid.gov/pdf_docs/PNACE007.pdf. Accessed Jan 1997.

5. American society of Hospital pharmacists. ASHP Guidelines on Medication Use Evaluation. Am J Health Syst Pharm. 1996;53:1953-5.

6. Academy of managed care pharmacy, Concepts in Managed Care pharmacy: drug use evaluation. 1998. Available

at: http://depts.washington.edu/exphasmed. Accessed Jan 2009.

7. DACA: National Drug List of Ethiopia, Drug Administration and control Authority of Ethiopia, Addis Ababa, 2002. Available at: http://apps.who.int/medicinedocs/documents/s16179 e/s16179e.pdf. Accessed July 2002.

8. Richard D. Howland \& Mary J. Mycek. Principles of antimicrobial. In: Lippincott's illustrated Reviews: pharmacology. $3^{\text {rd }}$ ed. Philadelphia; 2006: 382-383.

9. Von Gunten V, Troillet N, Beney J, Boubaker K, Luthi JC, Taffe P, Reymond JP. Impact of an interdisciplinary strategy on antibiotic use: a prospective controlled in three hospitals. J. Antimicrob. Chemother. 2005;55;362-83.

10. Raveh D, Muallem Zilcha E, Greenberg A, Wienerwell Y, Schlesinger Y, Yinnon AM. Prospective drug utilization evaluation of three broad spectrum antimicrobials: Cefepime, Piperacillin- tazobactam and meropenem. QJ Med. 2006;99:397-476. 
11. Rehana HS, Nagarani MA, Rehan M. A study on the drug prescribing pattern and use of antimicrobial agents at a tertiary care teaching hospital in Eastern Nepal. Indian J Pharmacol. 1998;30:175-80.

12. Moddley P. Moodley D, Sturm Aw: ciprofloxacin resistant Neisseria gonorrhoea in South Africa Int $\mathrm{J}$ Antimicrobial Agents. 2004;24:192-3.

13. Richard W Druckenbrod, Todd king, J. Edward Herring: Evaluation of potentially unnecessary ciprofloxacin use in Long-Term care facilities, central North Carolina.

14. American society of hospital pharmacists: Ciprofloxacin drug utilization review and prospective drug use evaluation. Available at: http:// www.theannals.com/cgi/content /abstract /24/1/82. Accessed Jan 2009.

15. Hammer man A. Green berg A: Drug use evaluation of ciprofloxacin, impact of educational efforts on appropriateness of use, Journal of Clinical Pharmacy and therapeutics. 1997;22(1):415-20.
16. Dydek GJ, Souney PF, Matthews SJ: Drug use evaluation of ciprofloxacin in the treatment of urinary tract infections in hospitalized patients. Availabale at: http/www.ncbi.nlm.nih.gov/pubmed/1011671. Accessed Jan 2009.

17. Abdel Muemin IA. Investigation of drug use in health centers in Khartoum state. Sudan Medical Journal. 1999;37:21-6.

18. Deriba L, Worku F, Girma T. Drug use evaluation of Co- trimoxazole preventive therapy for people living with HIV/AIDS in Jimma University Specialized Hospital, South West Ethiopia, 2007 Jan.

19. Abier Hamami. RPH. CPHQ. Determination of sample size for drug use evaluation, 2007. Available at

http://www.Pharmacorner.com/default.asp/action=pa rticle and ID+136. Accessed Jan 2009.

doi:10.5455/2319-2003.ijbcp20140222

Cite this article as: Biru TT, Defersha AD, Gelaw BK, Tegegne GT. Drug utilization review of ciprofloxacin in the outpatient department of Boru Meda Hospital, South Wollo Zone, Amhara Region, Ethiopia. Int J Basic Clin Pharmacol 2014;3:171-8. 